\title{
Status of the Smithsonian Microbeam Standards 2017 With a Discussion of the Venerable VG-2 Basalt Glass
}

\author{
Timothy R. Rose ${ }^{1}$ and Cathleen Brown ${ }^{1}$ \\ 1. Department of Mineral Sciences, Smithsonian Institution, Washington, D.C., USA
}

In 1980, the compositions of a set of microbeam reference materials were published [1] and made available to the world. These mostly mineral and natural glasses were characterized by classical wetchemical analysis by staff of the Department of Mineral Sciences of the Smithsonian Institution (SI) and named the Smithsonian Microbeam Standards (SMS). Additional carbonates, synthetic rare-earth element phosphates and trace element doped synthetic glasses were added to the collection bringing the total number of currently available samples to 57. To date more than 1300 requests have been filled with over 20,000 individual samples distributed to laboratories worldwide free of charge. Whereas a few of these are no longer available because of very limited quantity, most have quantities sufficient for distribution for many decades at the current demand in aliquots of sufficient size for electron beam microanalysis reference mounts. However, in the last ten years there has been about a threefold increase in the number of SMS distributed (see figure 1). The reason for this increase is not clear. Many requests are for labs with new instrumentation including more frequently for quantification using energy dispersive x-ray spectroscopy. More requests are also coming from laser ablation labs for which only some of the SMS are in sufficient quantity to be appropriate. Laboratories with heavily used mounts in need of replacement are encouraged to request replacement samples. The Corning glass archaeological reference samples, doped with selected trace elements, exist in reasonably large enough quantities to be suitable for destructive analytical methods.

The wet chemically determined composition of sample NMNH 111240-52, more commonly known as VG-2, has been under scrutiny from the beginning. VG-2, from the Juan deFuca ridge, was selected from the SI abyssal volcanic glass collection that was amassed by William Melson and Tim O'Hearn beginning in 1969 [2]. It is widely known that the original $\mathrm{MgO}$ value published by Jarosewich et al. [1] is likely too low. Along with another basalt glass, NMNH 113498-1, also known as A-99, VG-2 was chosen for a 1979 interlaboratory study involving three electron microprobe (EPMA) labs at MIT, USGS-Reston and the SI [3]. It was concluded at the time that the wet chemical analysis and the EPMA analyses were "in excellent agreement." This conclusion was based on an extremely limited data set of only 6 total analyses of VG-2 and 9 of A-99. Melson et al. agreed with this assessment in 2002 [2]. Nevertheless, the average value for $\mathrm{MgO}$ in VG-2 from the three labs is 0.21 weight per cent higher than the wet analysis (6.94 vs 6.71), whereas the average for A-99 is different by only 0.06 weight per cent. More recently two studies have evaluated the composition of VG-2 by repeated EPMA analyses. Gale et al [4] determined a set of suggested correction factors, based on 195 analyses of VG-2, to use on volcanic glass compositions determined by EPMA at the Smithsonian. They determined a composition of 6.95 weight percent $\mathrm{MgO}$. Helz et al. [5] found the same value of 6.95 weight per cent $\mathrm{MgO}$ to be appropriate for correction of a volcanic glass data set that originally used $\mathrm{VG}-2$ as the $\mathrm{MgO}$ reference. This value was determined by blind reanalysis of selected grains from the original study instead using A-99 for the $\mathrm{MgO}$ reference in two different labs (SI and USGS-Reston). In spite of the problem with magnesium, many laboratories have adopted VG-2 as a valuable sulfur reference sample. Figure 2 shows a chronology of the published $\mathrm{S}$ composition of VG-2. Detailed information about these analyses can be found on the SI data sheet for VG-2. 
More information about the SMS can be found at: http://mineralsciences.si.edu/facilities/standards.htm

References:

[1] E Jarosewich, JA Nelen and JA Norberg, Geostandards Newsletter, 4(1) (1980), p. 43.

[2] WG Melson, T O’Hearn, and E Jarosewich, Geochem. Geophys. Geosyst., 3 (2002), p. 11.

[3] E Jarosewich, AS Parkes and LB Wiggins, Smithsonian Contributions to the Earth Sciences, 22 (1980), p.56.

[4] A Gale et al, Geochem. Geophys. Geosyst. 14(3) (2013), p. 489.

[5] RT Helz, DA Clague, LG Mastin, and TR Rose, USGS Open-File Report 2014-1090 (2014).

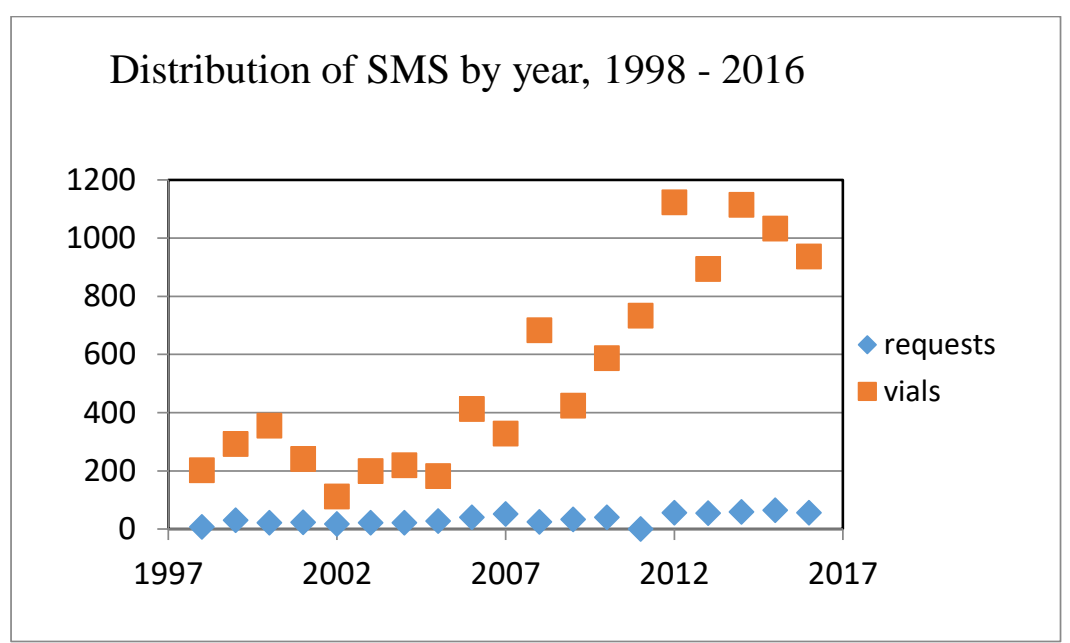

Figure 1. Number of SMS requests and samples distributed.

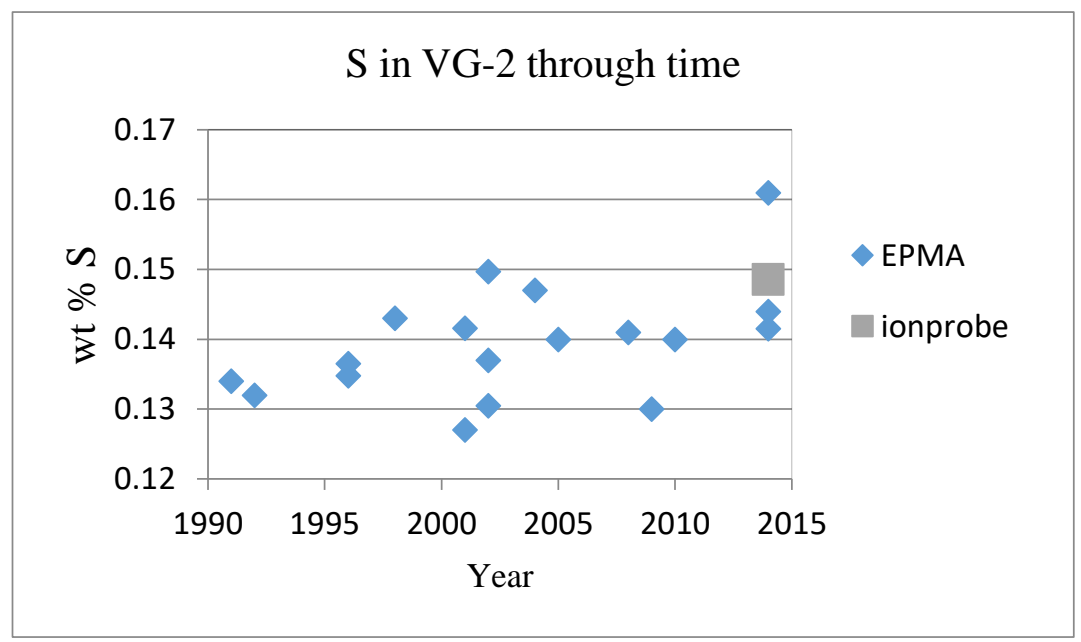

Figure 2. Analyses of S in VG-2 compiled from the literature. 